\title{
El papel de los agentes en las políticas agrícolas: intenciones y realidad
}

\section{Martine Dirven \\ Unidad de Desarrollo \\ Agrícola, CEPAL}

Las políticas sectoriales hacen supuestos explícitos e implícitos sobre el comportamiento y las capacidades de los agentes (como las respuestas dinámicas a las señales de mercado, la asistencia impulsada por la demanda, los esfuerzos colaborativos, la participación en el financiamiento) que a nuestro juicio son poco realistas. Por esta falta de realismo, políticas que intentan ser neutras resultan a menudo muy excluyentes. En ellas no se da suficiente importancia a las peculiaridades del sector - con sus altos riesgos climáticos, biológicos y comerciales y su lenta adaptación-, ni al hecho de que quienes toman decisiones en el agro están hoy mayoritariamente en inferioridad de condiciones, por sus ingresos por debajo de la línea de pobreza, su insuficiente preparación, sus tradiciones basadas en siglos de vida en condiciones precarias y su ubicación geográfica en zonas marginales, alejadas de la infraestructura y de un mínimo de servicios y fuentes de información. Estas personas tienen escaso e imperfecto acceso a los mercados que, según el modelo imperante, deben regir las decisiones y la (re)distribución de los factores de producción. A nuestro juicio, esto explica el dinamismo parcial y menor del esperado que ha exhibido el sector tras las reformas en pro de la liberalización de los mercados y la apertura externa en el región. Frente a los resultados de la aplicación del nuevo modelo, cabe preguntarse si América Latina puede permitirse un desarrollo que deje fuera a más de la mitad de sus productores agrícolas, y también cuáles son las alternativas y cuáles los costos y beneficios productivos, monetarios, sociales, espaciales y otros de cada una de ellas. El artículo bosqueja los cambios en las políticas y los resultados a nivel agregado, resume los argumentos que suelen aducirse para explicar el desempeño agrícola en la región, y propone un segundo conjunto de explicaciones basadas en una descripción de los agentes y las respuestas que cabe esperar de ellos, contrastando estas últimas con el carácter supuestamente neutro de las políticas. 


\section{I}

\section{Introducción}

Varios autores, entre ellos Schejtman (1982), han subrayado la heterogeneidad de los productores agrícolas y, por lo tanto, la necesidad de formular políticas diferenciales para obtener los resultados perseguidos. Esto se hizo en el pasado, en un contexto de Estado paternalista, con políticas que en parte eran dictadas desde arriba y, por supuesto, desde la capital.

Más recientemente, pero en la misma línea y con bastante optimismo, De Janvry y Sadoulet (1993) concluyen que el momento actual debiera ser especialmente propicio para el desarrollo rural, ya que hoy se entiende mejor la dinámica de la pobreza rural, la lógica de las instituciones agrarias y la economía política de las reformas.

Sin embargo, a nuestro juicio, la situación actual apunta más bien a lo contrario: se hace hincapié en políticas macroeconómicas "neutras" en lo sectorial, con bastante indefinición en cuanto a la cabida de políticas sectoriales dentro del "modelo", con un énfasis incipiente en definiciones de políticas de fomento a nivel regional y local, y uno mayor en acciones y servicios impulsados por la demanda, de modo que son los agentes los que toman la iniciativa en esta demanda, que en muchos casos debería ser de carácter grupal.

En general se esperaba que el sector agrícola, que había sido gravado fuertemente durante los cerca de treinta años de política de sustitución de importaciones ${ }^{1}$ ahora aprovecharía las ventajas comparativas de los recursos naturales de la región y mostraría todo su potencial de desarrollo y de crecimiento exportador. Sin embargo, hasta el momento su desempeño ha sido más bien decepcionante.

Empiezan a surgir análisis microeconómicos ${ }^{2}$ que llegan a la conclusión de que las políticas neutras, además de favorecer a los bienes transables exportables y -menos previsiblemente- a varios de los no transables (CEPAL, 1997a), también han favorecido a algunos grupos más que a otros.

Pensamos que, complementando un estudio reciente acerca de la influencia de los agentes sobre las políticas (Binswanger y Deininger, 1997), un análisis que parta de la realidad y de la capacidad de respuesta de los distintos agentes a las diferentes políticas debiera contribuir a explicar por qué el sector en su conjunto ha tenido este desempeño menor al previsto con el modelo neoliberal.

Queremos, entonces, después de examinar los cambios introducidos en las políticas y las indicaciones sobre el desempeño del sector, esbozar aquí una reflexión que, partiendo de las distintas características socioeconómicas de los productores y de las diferencias en la intensidad del uso de los factores en los distintos productos silvoagropecuarios, indique cómo les afectan las señales de precios, políticas e instrumentos o cómo es más probable que respondan a ellas.

\section{II}

\section{Las políticas económicas después de 1980}

Desde mediados de los años ochenta América Latina ha vivido cambios sin precedentes desde una estrategia de desarrollo volcada hacia los mercados internos con fuerte intervención del sector público a una orientada al exterior, con mercados desregulados, mucho control fiscal y el sector privado como agente prota-

La autora agradece la colaboración de Mónica Arellano, Asistente de Investigación.

${ }^{1}$ Véase, entre otros, Krueger, Schiff y Valdés, 1990. gónico del desarrollo. Se esperaba que esta nueva orientación llevara a un crecimiento rápido, eficiente y libre de las distorsiones anteriores.

Sin embargo, no se puede hablar realmente de la existencia de "un" nuevo modelo de desarrollo en la región. Existe actualmente un cuasi consenso en torno a la necesidad de mantener los equilibrios macroeco-

\footnotetext{
${ }^{2}$ Respecto al sector industrial, véase por ejemplo, Peres, coord., 1998.
} 
nómicos; de llevar a cabo una apertura comercial y financiera; de limitar la acción del Estado en actividades productivas, y de desregular los mercados. El consenso es menor en cuanto a la necesidad de regular y supervisar el sistema bancario; a la secuencia en que debieran darse la reforma comercial, la liberalización financiera y la política cambiaria y la necesidad previa de contar con un marco institucional financiero; a la necesidad de políticas de promoción de exportaciones y de desarrollo sectorial y tecnológico; a las privatizaciones, y al contenido y alcance de las reformas laborales (CEPAL, 1995).

La reducción de la inflación ha sido sin duda uno de los hitos exitosos. Otras variables macroeconómicas claves para la competitividad de un sector transable como el agrícola han sido abordadas con menos efica- cia; como resultado, gran parte de las monedas nacionales de la región están sobrevaluadas y las tasas de interés son muy altas. A raíz de esta situación y de una capacidad de reacción de los distintos agentes menor que la prevista, el decenio de 1990, aun antes de la crisis asiática, tendía a concluir con una tasa de crecimiento media inferior a la que caracterizó el período de sustitución de importaciones y una distribución del ingreso similar o aún más concentrada que al inicio de los años ochenta.

Desafortunadamente, la división en dos bandos de los que están a favor del modelo neoliberal, por un lado, y los que tienen serios reparos a él, por otro, no ha permitido aún un análisis desapasionado y un debate constructivo sobre este desempeño inferior al esperado.

\section{III}

\section{Las políticas agrícolas anteriores y posteriores a 1980}

Generalizando, se puede afirmar que en los años cincuenta a ochenta hubo políticas sectoriales en favor de la agricultura con el afán, entre otros, de neutralizar otras políticas con orientación industrial y urbana. Según Krueger, Schiff y Valdés (1990), el conjunto de estas medidas habría sido desfavorable para la agricultura. Según estos autores, las políticas macroeconómicas tuvieron un fuerte impacto negativo que no logró ser compensado por las políticas sectoriales a favor del sector agrícola. ${ }^{3}$ Además, los subsidios a los insumos, al agua, al crédito y a las tarifas de diversos servicios favorecieron a quienes los usaban más, es decir, a los medianos y grandes productores empresariales (Gómez Oliver, 1994).

El neoliberalismo postula básicamente que el mercado - en condiciones de competencia e información perfectas - es el que mejor asigna los recursos productivos entre varios usos y los hace fluir hacia donde están las ventajas comparativas del país y del agente económico. El sector privado se transforma en el agente central de cambio. ${ }^{4}$

\footnotetext{
${ }^{3}$ Se utiliza "agrícola" en este artículo para simplificar, pero en general se refiere más bien a lo agropecuario y a veces también a lo silvícola y pesquero.

${ }^{4}$ El Foro Iberoamericano de Agricultura (Santiago, agosto de 1996) lo expresó así en la Declaración de Santiago: “... reconoce en los
}

Las políticas de apertura (rebaja de aranceles y eliminación de trabas a la importación y exportación, como cuotas y licencias) son, por lo tanto, una consecuencia lógica del modelo neoliberal. En cambio, la gran importancia asignada al tema de las exportaciones no lo es; de hecho, deriva de la necesidad de equilibrar la balanza de pagos. Efectivamente, si bien para aprovechar cabalmente las rentabilidades en la industria es indispensable — en economías medianas a pequeñas - tener una estrategia exportadora, no sucede lo mismo en la agricultura, donde las economías de escala son a menudo inexistentes, ni por lo general tampoco en la agroindustria. Por lo tanto, el desarrollo basado en un mercado interno, que demanda calidad y

\footnotetext{
emprendedores productivos privados los agentes centrales de cambio, ya sean éstos empresas privadas de propiedad individual, cooperativas, sociedades, comunidades o de cualquier otra forma, y que el Estado debe jugar un papel facilitador, que tiene por objetivo fortalecer una base de agentes emprendedores sólida, amplia y creativa, para beneficios de quienes asumen los riesgos de emprender y de los países como un todo."; matizado por: "Reafirmar el compromiso del Estado con el apoyo a la agricultura, en especial a la agricultura campesina familiar y las asociaciones cooperativas, como la respuesta más efectiva y realista para un segmento significativo de productores agrícolas medianos y pequeños, los que por razones económicas, sociales, ambientales y culturales, tienen en esta opción una posibilidad de desarrollo."
} 
está abierto a la competencia externa, es absolutamente legítimo. 5

En los años noventa, junto con desplegar esfuerzos encaminados a la liberalización de los mercados y a la apertura al exterior se ha avanzado hacia procesos de descentralización; nuevas instancias de consulta y participación; incentivos para la concertación entre distintos agentes; nuevas formas de asociación Estado-agentes privados; transferencia de funciones desde el Estado a agentes privados $\mathrm{u}$ organizaciones no gubernamentales, y fomento de políticas transversales (el mejoramiento de la educación general, por ejemplo) en vez de sectoriales.

En consecuencia, el aparato público para la agricultura y el medio rural está actualmente en pleno proceso de transformación, de redefinición y justificación de sus ámbitos de acción y de revisión de sus relaciones con la sociedad civil, mientras esta última pasa de ser beneficiaria a ser protagonista y, como las demás reparticiones públicas, se esfuerza por aumentar su eficiencia. Sin embargo, para que la sociedad civil pueda convertirse en protagonista, es necesario que se cumplan una serie de requisitos, tema al cual regresaremos luego.
En general, el Estado ha reducido sus actividades. Como resultado, en el área agrícola, que se caracteriza por sus altos riesgos y la gran dispersión geográfica de sus agentes, se han generado vacíos de los que no se ha hecho cargo aún cabalmente el sector privado, el que no los asumirá sin compensación en áreas poco rentables. Estos vacíos han tenido efectos adversos, en especial para los pequeños y medianos productores, en el acceso al crédito, a la asistencia técnica agrícola, a los mercados y a los centros de acopio, y también en la investigación orientada al mejoramiento genético de las semillas y el conjunto de tecnologías destinado a la agricultura de pocos recursos y tierras marginales. Las organizaciones no gubernamentales, cada una con su enfoque especializado y de conformidad con las directrices de las entidades que las financian, han ocupado algunos de los espacios dejados por el Estado. Sin embargo, sus actividades, en general a pequeña escala y muy localizadas, han creado dificultades de coordinación, retroalimentación y difusión aún mayores que cuando el desarrollo rural estaba a cargo de distintas reparticiones del Estado.

Simplificando y sintetizando mucho, en el cuadro 1 hemos tratado de reseñar los grandes rasgos de los cambios en las políticas agrícolas.

\section{IV}

\section{Los resultados esperados y los obtenidos}

Teóricamente, las políticas de ajuste, de liberalización de los mercados internos y de apertura externa - lo que implicaba también la eliminación de los fuertes gravámenes que pesaban sobre la agricultura-, debían generar un crecimiento del sector en su conjunto mayor que el registrado históricamente (3\% anual entre 1950 y 1980 , y $3.5 \%$ entre 1970 y 1980). Asimismo, debían llevar a una reorientación de los recursos para aprovechar mejor las ventajas comparativas de la región (atenuadas por las políticas de intervención de los países

\footnotetext{
${ }^{5}$ Como anécdota: terminados los estudios hechos por la Corporación Colombiana Internacional sobre las posibilidades de colocar frutas tropicales en los mercados externos, se descubrió que el mercado interno colombiano tenía una demanda insatisfecha de tales productos tres o cuatro veces mayor que el potencial externo estimado en dichos estudios (IICA, 1996).
}

desarrollados). Todo ello debía dar un fuerte impulso a la agricultura de exportación en desmedro de los cereales básicos y de los cultivos poco transables — productos de consumo excesivamente local o demasiado perecibles o cuyo precio no compensa los costos de transporte-, que representan el grueso de la producción tradicional campesina.

Quince años después de iniciarse los procesos de reforma económica —aunque para el sector agrícola en muchos países éstos se iniciaron sólo en los años noventa- se puede decir que efectivamente está en marcha una reestructuración productiva en la dirección prevista (gráfico 1); pero ésta se había iniciado ya en los años setenta, es decir, antes de las reformas. Varios otros efectos de los procesos de reforma no están claros aún. Lo que sí está claro es que, hasta la fecha, los resultados económicos agregados del sector han sido menos alentadores de lo esperado por los que impulsaron las reformas. 
CUADRO 1

\section{América Latina y el Caribe: Grandes rasgos de los cambios en las políticas agrícolas}

Políticas de los años cincuenta a ochenta

Fuerte centralismo estatal

Planificación y financiamiento por parte del Estado de bienes y servicios "públicos"

Preocupación por la seguridad alimentaria del país y, por lo tanto, investigaciones orientadas al aumento de la producción y rendimiento de alimentos básicos (arroz, trigo, maíz, papas, frijoles, lentejas, etc.)

Asistencia técnica gratuita, con programas elaborados por entidades estatales con poca o nula participación de los usuarios

Crédito subsidiado a través de líneas de crédito especiales para el sector

Reformas laborales que llevaron a la monetización de la economía rural y a la desaparición paulatina de sistemas de inquilinaje, mediería, huasipunguismo, etc. y al reemplazo de esta mano de obra por asalariados permanentes y sobre todo temporales

Controles de los precios de los alimentos básicos para frenar la inflación y mantener salarios mínimos bajos, con miras a incentivar la industrialización

Tipos de cambio favorables a las importaciones

Aranceles altos en general pero con un amplio abanico de tasas

Impuestos a las exportaciones agrícolas

Programas de reforma agraria destinados a disminuir la ineficiencia de sistemas productivos muy heterogéneos (con abundancia de tierra y capital para algunos pocos agentes y abundancia de mano de obra para los demás), y sobre todo a disminuir las posibilidades de desórdenes sociales en el campo

\section{Políticas actuales}

Descentralización política, institucional y financiera orientada a responder a proyectos locales

Orientación hacia la demanda y la cofinanciación de las inversiones en infraestructura y servicios

Orientación hacia la demanda y la cofinanciación de la investigación tanto en los institutos públicos de investigación como en las universidades $^{\mathrm{a}}$

Asistencia técnica privada y pagada en respuesta a la demanda de los productores y después de la presentación de un proyecto a concurso cuando hay un subsidio involucrado

Eliminación de líneas de crédito específicas para el sector agrícola, de bancos de fomento y de la obligación de los bancos privados de prestar cierto monto de la cartera al sector

Desregulación del mercado de trabajo, aunque para la agricultura siempre hubo disposiciones menos estrictas y dificultades para fiscalizar su cumplimiento

Liberalización de los precios

En principio, tipos de cambio de equilibrio

Disminución de los aranceles y tasa uniforme, con algunas excepciones, en especial para productos agrícolas

Eliminación de los impuestos y otras trabas a las exportaciones

Reemplazo de los programas de reforma agraria por compras a través del mercado de tierras, asistidas por crédito y/o subsidio para los pequeños productores; formalización de la propiedad a través del otorgamiento de títulos ${ }^{\mathrm{a}}$

Fuente: Elaboración propia.

a Véase mayores detalles en las apéndices 1 y 2.

Así, si bien la crisis de los años ochenta golpeó menos a la agricultura que al resto de la economía, el crecimiento medio anual del sector - al igual que el de la economía en su conjunto- no se ha recuperado plenamente: fue de $2.5 \%$ entre 1991 y $1997,{ }^{6}$ con fuertes fluctuaciones de un año a otro (cuadro 2).

${ }^{6}$ Entre 1990 y 1995 hubo grandes mejoras en los rendimientos medios, que aumentaron a una tasa de $3.3 \%$ anual, tras haberlo hecho al $1.3 \%$ por año en el decenio de 1980. En cambio, el área cultivada, que había crecido en los años ochenta, disminuyó en $2.2 \%$ anual (FAO, 1996, p. 170).
Las exportaciones agrícolas aumentaron en volumen a un ritmo relativamente vigoroso en 1980-1996 (3.1\% anual), pero en valor su crecimiento fue débil hasta 1993, debido a la caída de los precios internacionales. A lo largo de todo el período, el crecimiento anual del valor de las exportaciones fue de $2.1 \%$ (gráfico 2). La participación de productos no tradicionales en las exportaciones agrícolas (como frutas y hortalizas frescas tropicales o de clima templado) ha aumentado y ellos representan actualmente cerca del $25 \%$ de esas exportaciones. En hectáreas sembradas y generación de empleo, sin embargo, la importancia de los productos no tradicionales es mucho menor. 
CUADRO 2 América Latina y el Caribe (23 países): Producto interno bruto de
la agricultura, silvicultura, caza y pesca y PIB total menos PIB de la
agricultura, silvicultura, caza y pesca

(Tasas anuales de crecimiento sobre la base de cifras en dólares a precios constantes)

\begin{tabular}{|c|c|c|c|c|c|c|c|c|c|c|c|c|c|c|c|}
\hline \multicolumn{9}{|c|}{ Tasa de crecimiento anual $1971-1980>$ promedio } & \multicolumn{7}{|c|}{ Tasa de crecimiento anual $1971-1980<$ promedio } \\
\hline & $\begin{array}{l}1991- \\
1997\end{array}$ & $\begin{array}{l}1981- \\
1990\end{array}$ & $\begin{array}{l}1971- \\
1980\end{array}$ & & $\begin{array}{l}1991- \\
1997\end{array}$ & $\begin{array}{l}1981- \\
1990\end{array}$ & $\begin{array}{l}1971- \\
1980\end{array}$ & & $\begin{array}{l}1991- \\
1997\end{array}$ & $\begin{array}{l}1981- \\
1990\end{array}$ & $\begin{array}{l}1971- \\
1980\end{array}$ & & $\begin{array}{l}1991- \\
1997\end{array}$ & $\begin{array}{l}1981- \\
1990\end{array}$ & $\begin{array}{l}1971- \\
1980\end{array}$ \\
\hline \multirow{5}{*}{ 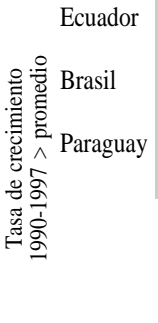 } & $\begin{array}{c}3.2 \\
(3.6)\end{array}$ & $\begin{array}{c}4.2 \\
(1.4)\end{array}$ & 3.0 & Rep. Dominicana & $\begin{array}{c}4.0 \\
(4.8)\end{array}$ & $\begin{array}{c}0.4 \\
(2.9)\end{array}$ & 3.4 & Chile & $\begin{array}{c}5.0 \\
(8.2)\end{array}$ & $\begin{array}{c}5.6 \\
(2.8)\end{array}$ & 2.2 & Guyana & $\begin{array}{c}9.5 \\
(6.6)\end{array}$ & $\begin{array}{c}-2.5 \\
(-3.1)\end{array}$ & 0.9 \\
\hline & $\begin{array}{c}3.0 \\
(3.0)\end{array}$ & $\begin{array}{c}2.5 \\
(1.5)\end{array}$ & 4.7 & Bolivia & $\begin{array}{c}3.7 \\
(4.2)\end{array}$ & $\begin{array}{c}1.7 \\
(-0.1)\end{array}$ & 4.1 & Perú & $\begin{array}{l}4.8 \\
(5.6)\end{array}$ & $\begin{array}{c}2.2 \\
(-1.4)\end{array}$ & -0.6 & Uruguay & $\begin{array}{c}4.2 \\
(4.1)\end{array}$ & $\begin{array}{l}0.2 \\
(-)\end{array}$ & 0.6 \\
\hline & $\begin{array}{c}2.5 \\
(2.8)\end{array}$ & $\begin{array}{l}4.0 \\
(2.7)\end{array}$ & 6.7 & Guatemala & $\begin{array}{c}2.8 \\
(4.5)\end{array}$ & $\begin{array}{l}1.3 \\
(0.8)\end{array}$ & 4.7 & Nicaragua & $\begin{array}{l}4.5 \\
(1.8)\end{array}$ & $\begin{array}{c}2.9 \\
(-1.8)\end{array}$ & -0.1 & Jamaica & $\begin{array}{l}2.6 \\
(0.1)\end{array}$ & $\begin{array}{c}0.9 \\
(2.3)\end{array}$ & 0.9 \\
\hline & & & & & & & & Honduras & $\begin{array}{c}3.4 \\
(3.8)\end{array}$ & $\begin{array}{c}2.7 \\
(2.3)\end{array}$ & 2.7 & Argentina & $\begin{array}{c}2.4 \\
(5.7)\end{array}$ & $\begin{array}{c}1.3 \\
(-0.9)\end{array}$ & 2.1 \\
\hline & & & & & & & & Costa Rica & $\begin{array}{c}2.6 \\
(3.6)\end{array}$ & $\begin{array}{c}3.1 \\
(2.1)\end{array}$ & 2.6 & & & & \\
\hline \multirow{4}{*}{ 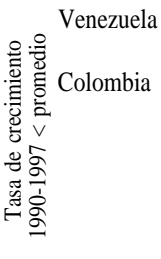 } & $\begin{array}{l}1.5 \\
(3.4)\end{array}$ & $\begin{array}{c}2.0 \\
(-0.9)\end{array}$ & & México & $\begin{array}{c}1.6 \\
(2.9)\end{array}$ & $\begin{array}{c}1.2 \\
(1.9)\end{array}$ & 3.4 & Panamá & $\begin{array}{c}1.6 \\
(5.1)\end{array}$ & $\begin{array}{c}2.9 \\
(1.2)\end{array}$ & 1.3 & $\begin{array}{l}\text { Trinidad } \\
\text { y Tabago }\end{array}$ & $\begin{array}{c}1.5 \\
(2.5)\end{array}$ & $\begin{array}{c}0.1 \\
(-2.7)\end{array}$ & -3.0 \\
\hline & $\begin{array}{c}1.4 \\
(4.6)\end{array}$ & $\begin{array}{c}3.0 \\
(3.9)\end{array}$ & 4.4 & & & & & & & & & El Salvador & $\begin{array}{l}1.2 \\
(6.0)\end{array}$ & $\begin{array}{l}-1.4 \\
(-0.2)\end{array}$ & 2.4 \\
\hline & & & & & & & & & & & & Barbados & $\begin{array}{l}-1.2 \\
(0.9)\end{array}$ & $\begin{array}{l}-2.4 \\
(1.3)\end{array}$ & -1.1 \\
\hline & & & & & & & & & & & & Haití & $\begin{array}{l}-6.4 \\
(-1.4)\end{array}$ & $\begin{array}{c}-0.2 \\
(-0.7)\end{array}$ & 1.5 \\
\hline
\end{tabular}

Fuente: Elaborado sobre la base de cifras proporcionadas por la División de Estadística de la CEPAL.

a Las cifras sombreadas corresponden a un crecimiento del PIB agrícola mayor que el promedio regional (sin Brasil) y las cifras entre paréntesis corresponden a la tasa de crecimiento anual en dólares constantes del PIB total menos el PIB agrícola. Tasa media de crecimiento anual del PIB agrícola de la región :

\begin{tabular}{ccc} 
& Sin Brasil & Con Brasil \\
$1991-1997$ & 2.3 & 2.5 \\
$1981-1990$ & 1.8 & 2.0 \\
$1971-1980$ & 2.9 & 3.5 \\
Tasa media de crecimiento anual del PIB total menos el PIB agrícola \\
\multicolumn{4}{r}{ Sin Brasil } & Con Brasil \\
$1991-1997$ & 4.1 & 3.7 \\
$1981-1990$ & 0.9 & 1.1
\end{tabular}

En cambio, las importaciones agrícolas, que habían disminuido fuertemente entre 1981 y 1987 (-5.8\% anual en valor), de allí en adelante retomaron su ritmo de crecimiento rápido iniciado a mediados de los años setenta (12.1\% anual en valor entre 1987 y 1996). Se trata esencialmente de productos básicos para la alimentación, como cereales y lácteos. A lo largo de todo el período 1980-1996, las importaciones agrícolas crecieron a un ritmo anual de $3.0 \%$ en volumen y de $3.9 \%$ en valor.

En la economía en su conjunto, el crecimiento a partir de los años noventa se ha basado en el uso bas- tante intensivo de capital y ha creado poco empleo; la diferencia entre los salarios de los trabajadores más calificados y de los no calificados ha aumentado, y la brecha entre los ricos y los pobres también. En general, el modelo parece ser excluyente para un grupo importante de la población y también en el sector agrícola (cuadro 3). Contrariamente a lo que ha acontecido en el área urbana, en el área rural los niveles de pobreza y los de indigencia posteriores a la crisis parecen estar estancados en cifras altas: 54 y $31 \%$ de los hogares rurales o 78.2 y 47.0 millones de personas, respectivamente, en 1997 (CEPAL, 1999). 
GRAFICO 1

\section{América Latina y el Caribe: Cambio en la estructura de producción}

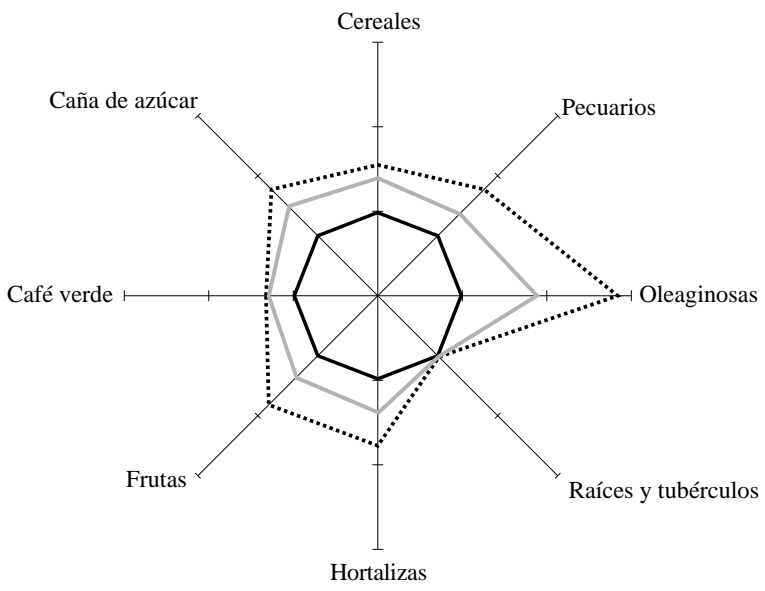

$1970-1975 \longrightarrow 1980-1985 \quad \ldots \ldots \ldots . . . .1990-1995$

Fuente: CEPAL/IICA (1997), con datos de la Organización de las Naciones Unidas para la Agricultura y la Alimentación (FAO).
GRAFICO 2

\section{América Latina y el Caribe: \\ Comercio agrícola}

(Base 1989-1991 = 100)

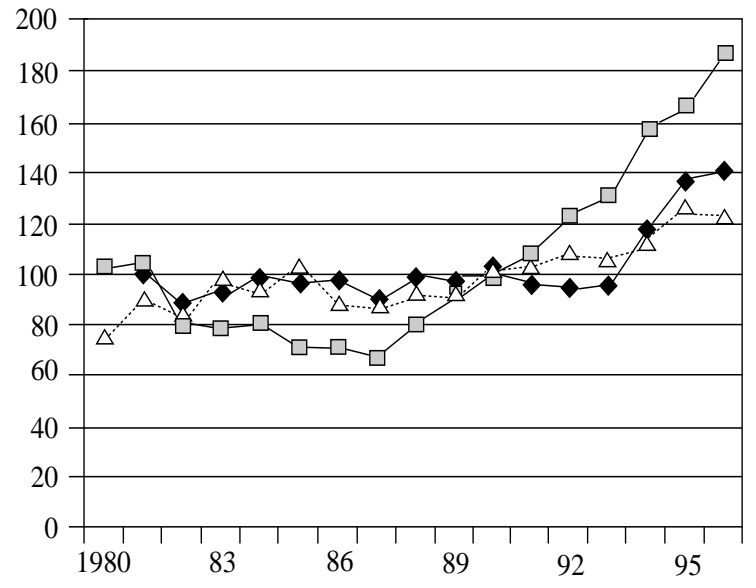

Exportaciones $-\square-$ Importaciones $\cdots \triangle \cdots$ Exportaciones (valor) (valor) (cantidad)

Fuente: FAO-Internet, diciembre de 1998.

CUADRO 3

América Latina: Cambios en la estructura de producción agrícola, 1970-1995

\begin{tabular}{|c|c|c|c|c|c|}
\hline Producción & $\begin{array}{c}\text { Sector } \\
\text { A }\end{array}$ & $\begin{array}{c}\text { Sector } \\
\text { B }\end{array}$ & Superficie & Productividad & $\begin{array}{l}\text { Intensidad de uso } \\
\text { de mano de obra }\end{array}$ \\
\hline Oleaginosas & $\mathrm{X}$ & & Aumentó fuertemente & Aumentó fuertemente & Poco intensivo \\
\hline Cereales & $\mathrm{X}$ & & Aumentó & Aumentó fuertemente & Poco intensivo \\
\hline Cereales & & $\mathrm{X}$ & Disminuyó & Estancada & De intensidad mediana \\
\hline Hortofruticultura & $\mathrm{X}$ & $\mathrm{X}$ & Aumentó & Aumentó & Intensivo \\
\hline Ganadería (carne) & $\mathrm{X}$ & $\mathrm{X}$ & Aumentó & Aumentó algo & Poco intensivo \\
\hline Ganadería (leche) & $\mathrm{X}$ & $\mathrm{X}$ & Aumentó & Aumentó & Intensivo \\
\hline $\begin{array}{l}\text { Pecuarios menores } \\
\text { (aves, cerdos) }\end{array}$ & $\mathrm{X}$ & & Poco importante & Aumentó & Intensivo \\
\hline Tubérculos y legumbres & & $\mathrm{X}$ & Estancada & Estancada & De intensidad mediana \\
\hline Bosques plantados & $\mathrm{X}$ & & Aumentó algo & Aumentó & Poco intensivo \\
\hline Bosques naturales & & $\mathrm{X}$ & Disminuyó & Estancada & - \\
\hline
\end{tabular}

Fuente: Elaboración propia.

a Sector A: preponderantemente moderno

Sector B: preponderantemente tradicional. 


\section{V \\ Interpretación de un desempeño agrícola más bien decepcionante}

Es importante recalcar que los que impulsaron las reformas habían identificado serias trabas al desarrollo - en particular para el sector agrícola — en el modelo anterior y, por ende, esperaban un crecimiento más dinámico que el histórico a raíz de las reformas.

Para entender el desempeño inferior al previsto, ${ }^{7}$ conviene recordar que los países aplicaron medidas de ajuste, de libre mercado y de apertura de distinta fuerza y escalonadas de distinta manera en el tiempo, ${ }^{8}$ con una serie de excepciones para el sector agrícola. En varios países, los mercados empezaron a liberarse sólo a fines de la década de 1980 o incluso más tarde (gráfico 3), mientras que en otros, después de un corto período de liberalización, se introdujeron nuevamente aranceles y medidas de apoyo a los precios y a la comercialización interna. ${ }^{9}$ De hecho, muchos de los cambios en la política agrícola, en vez de obedecer a una estrategia de desarrollo bien definida, tuvieron más bien el carácter de medidas especiales en respuesta a preocupaciones macroeconómicas o sectoriales de corto plazo y a demandas de los grupos de presión.

Las negociaciones comerciales tampoco obedecieron a estrategias definidas y los niveles consolidados en la ronda Uruguay (arancel teórico) resultaron en grandes dispersiones, incluso entre países que tienen

\footnotetext{
${ }^{7}$ La CEPAL está desarrollando dos proyectos interdisciplinarios de envergadura con el fin de analizar si fue la teoría o su aplicación lo que falló o si es demasiado temprano aún para ver todos los frutos de las reformas. Aquí indicaremos sólo a grandes rasgos las explicaciones que se perfilan en cuanto al sector agrícola.

${ }^{8}$ Entre otros autores, véase Ramos (1997).

${ }^{9}$ Fue el caso, entre otros, de Chile (con la reintroducción de poderes compradores después de la crisis de 1982 y la introducción de bandas de precios). En Colombia se introdujeron o reintrodujeron medidas como precios mínimos de importación, régimen de licencia previa, fondo de estabilización para el algodón, presencia más activa de las estaciones de acopio del Instituto de Mercadeo Agrícola (IDEMA), reapertura de líneas de crédito, rescate de la Caja Agraria, plan de choque tecnológico y seguro agropecuario. José Antonio Ocampo, Ministro de Agricultura de Colombia en 1993, justificó estas medidas por la trascendencia económica y sobre todo social del sector, y también porque, según afirmó textualmente, "ha fracasado la idea de que para el conjunto de la actividad agropecuaria, la mera reducción de los niveles de protección era lo único necesario para que el sector floreciera". (Colombia, Ministerio de Agricultura, 1993, pp. 3-7)
}

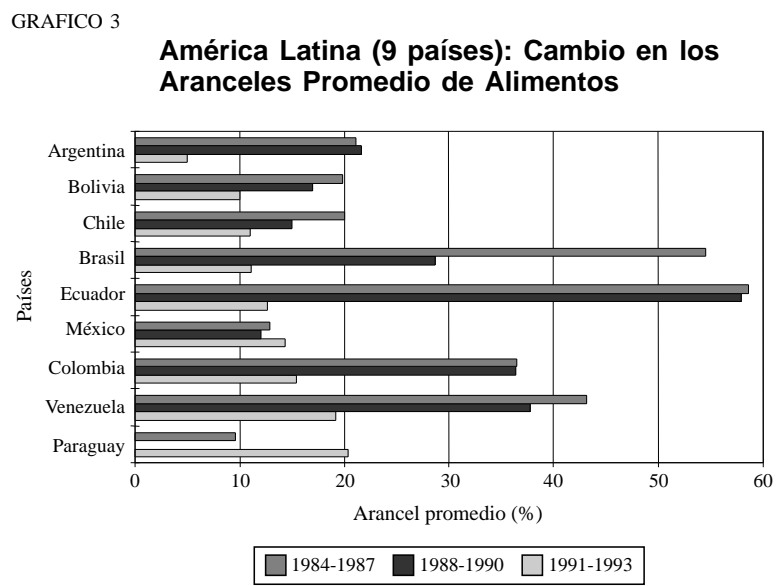

Fuente: CEPAL/IICA (1997), sobre la base de datos de la Conferencia de las Naciones Unidas sobre Comercio y Desarrollo (UNCTAD).

acuerdos bilaterales o multilaterales, y no configuraron toda la estructura de derechos correctivos para compensar las condiciones de competencia desigual. ${ }^{10}$

Además, durante gran parte de los últimos 15 años, los esfuerzos de apertura coincidieron con la vigencia de un mercado mundial deprimido, con tipos de cambio que en la mayoría de los países de la región primero estaban devaluados en exceso y luego sobrevaluados, ${ }^{11}$ con restricciones y encarecimiento del crédito, ${ }^{12}$ y con una caída del ingreso per cápita que

\footnotetext{
${ }^{10}$ Ilustran este punto los países andinos, que tienen un arancel externo común (entre 15 y 20\%) suplementado por franjas de precios o arancel flexible para un conjunto de productos agropecuarios. Sin embargo, Colombia negoció aranceles consolidados que fluctúan entre $15 \%$ y $332 \%$ con un promedio de $118 \%$; Ecuador negoció un arancel medio de 26\%; Venezuela consolidó la mayor parte de los productos agrícolas en $40 \%$, pero con algunas franjas que llevan el promedio a 59\%; y Bolivia consolidó de manera uniforme en 40\%, es decir tres veces el arancel normal (IICA, 1996).

${ }^{11}$ Comparando el tipo de cambio real vigente en 1996 con el de 1990, éste aumentó y por lo tanto favoreció las exportaciones en sólo seis países: Bolivia, Honduras, Nicaragua, Paraguay, Costa Rica (hasta 1995) y México (en 1994 y 1995). En todos los demás países disminuyó (CEPAL/IICA, 1997, sobre la base de datos de la CEPAL).

12 Comparando la tasa de interés real vigente en 1996 con la de 1992, ésta disminuyó en Chile, Honduras, Panamá, Perú, Venezuela, Brasil (a partir de 1994) y Costa Rica (a partir de 1993). En todos los demás países aumentó (CEPAL/IICA, 1997, sobre la base de datos de la CEPAL).
} 
causó un menor consumo interno de productos agropecuarios y un desplazamiento hacia productos menos caros, a menudo procedentes de la agricultura tradicional (como cereales, legumbres secas y tubérculos). Es probable que todos estos factores en conjunto hayan frenado la reestructuración esperada del sector más moderno. Y que el menor consumo y el reemplazo parcial de diversos servicios estatales por servicios privados equivalentes o mejores, sumados a la escasa capacidad de adaptación (por falta de recursos, conocimientos e información y exceso de riesgos) del sector tradicional campesino, hayan frenado también los posibles cambios en este sector, salvo algunas experiencias exitosas de coordinación vertical con agronegocios, en especial de agroexportación. En este contexto, las medidas reformistas han causado más bien fuertes tensiones entre los agricultores y las autoridades, las que a menudo - al igual que en los países desarrollados - se han resuelto mediante la reintroducción de disposiciones proteccionistas.
Merced a las políticas orientadas a la apertura comercial y al desarrollo que ha experimentado la infraestructura, la producción agrícola se ha vuelto más transable internacionalmente y el precio de los productos depende cada vez menos de la demanda y oferta microrregionales y nacionales (o de políticas arancelarias y de precios) y cada vez más de las fluctuaciones de la demanda y oferta externas. El efecto regulador sobre los ingresos de los productores que tenían las alzas (o bajas) de precio a causa de malas (o abundantes) cosechas locales, se deja sentir cada vez con menor fuerza. Frente a esta exposición creciente a los precios internacionales, el sector agrícola podrá sostenerse: i) si logra producir a precios competitivos internacionalmente y crear mecanismos para aminorar los riesgos u ofrecer ingresos suficientes para compensarlos y para retener a los productores por cuenta propia, los empleadores y los asalariados, y ii) si puede atraer las inversiones necesarias para seguir siendo competitivo en el plano internacional y también nacional.

\section{VI}

\section{Interpretación adicional}

Hasta ahora, la interpretación de las causas del desempeño agrícola observado es de corte bastante tradicional. A continuación, explicaremos por qué nos parece que varias de las innovaciones introducidas en las políticas sectoriales son más bien excluyentes si no van acompañadas de medidas para potenciar al sector.

\section{Los agentes y la realidad agrorrural de la región}

Un cálculo muy grueso de la población económicamente activa (PEA) de la región indica que hay unos 17 millones de productores agrícolas -empleadores y trabajadores por cuenta propia- en una PEA agrícola de unos 41 millones. Estos productores son los agentes económicos de los cuales se esperan respuestas a los estímulos de política. Dejaremos de lado a los 3 millones de empleadores - que, de alguna manera, debieran tener mayor capacidad de respuesta por contar con más capital y probablemente una mejor preparación- y nos concentraremos en los 14 millones de trabajadores agrícolas por cuenta propia (gráfico 4 y cuadro 4). Según las estadísticas de pobreza (CEPAL, 1999) el porcentaje de pobres entre los trabajadores por cuenta propia y la población rural total es similar, así que, grosso modo, un 55\% de esos 14 millones se halla por debajo de la línea de pobreza (y posiblemente también un tercio de ellos por debajo de la línea de indigencia), con todo lo que esto implica para la capacidad de acumular recursos de inversión, de pensar más allá del corto plazo, de asumir riesgos e innovar y de ver la actividad agrícola como una opción válida de largo plazo.

Si se añade a ello la falta de escolaridad —más acentuada aún entre quienes toman las decisiones, es decir, los mayores de 40-50 años-, las fallas y asimetrías del mercado de la información y las exigencias cada vez mayores de un mercado ágil, abierto y globalizado, es fácil entender que un grupo muy importante de los agentes se encuentre en condiciones de franca desventaja o de indiferencia ante los supuestos estímulos de política. Hablamos de indiferencia porque al no tener acceso al crédito, les es indiferente - de manera directa - si la tasa de interés sube o baja; y si primordialmente autoconsumen o utilizan insumos y bienes mayoritariamente nacionales, les atañe poco — directamente - si el tipo de cambio se deprecia o aprecia. 
GRAFICO 4

América Latina: Población económicamente activa, 1995

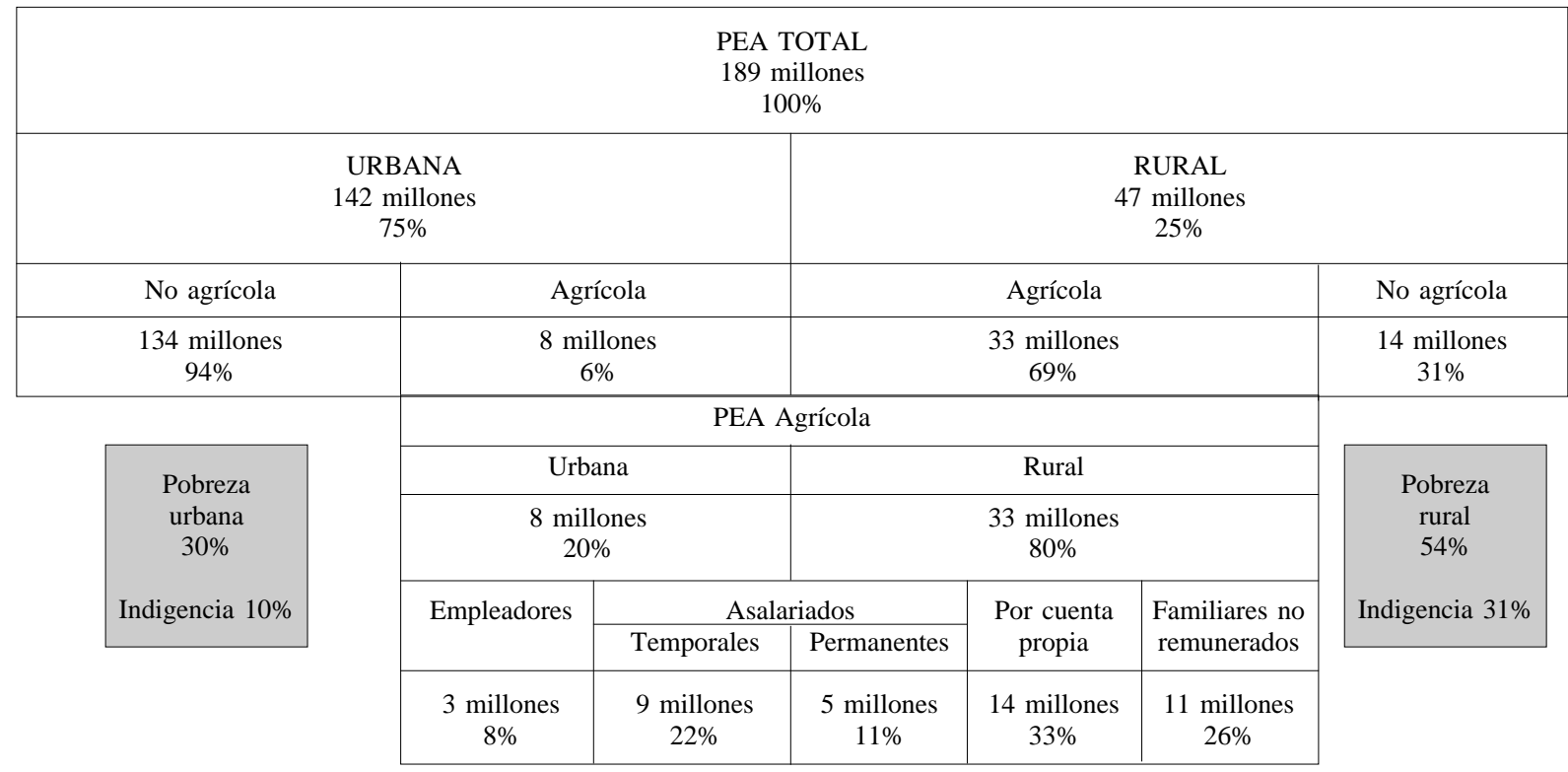

Fuente: Elaboración propia sobre la base de CELADE (1995 y 1996), FAO (1994), Klein (1992), encuestas de hogares de varios países efectuadas en 1992 y CEPAL (1999).

Además, los costos de transacción fijos y la existencia de mercados imperfectos (en especial de tierras, crédito y mano de obra) explican la aparente falta de reacción a cambios en los precios de grandes grupos de productores agrícolas constreñidos por su escasez de recursos. En efecto, los costos de transacción fijos (información, organización, contratación) en los cuales es necesario incurrir para pasar de la producción de bienes de autoconsumo a la de bienes que se vendan en los mercados pueden ser muy altos, lo que obligaría a producir una cantidad mínima aunque la producción misma sea neutra en cuanto a escala. Además, los costos de transacción fijos quizás no sean recuperables a través de otras actividades, y si son muy altos, obligarían a participar varios años seguidos en un mercado dado. Por este motivo, el productor tal vez decida no incurrir en tales costos si no está seguro de poder participar en el mercado por el tiempo necesario. Ahora bien, a medida que la participación en los mercados es más riesgosa y que no existen mecanismos de seguro externos a la finca, los productores optarán por un grado de autosuficiencia alimenticia más allá de lo indicado por la magnitud de los costos de transacción. A esto se debe la estrategia de seguir produciendo cultivos para el autoconsumo incluso por pequeños productores que participan activamente en los mercados (De Janvry y Sadoulet, 1998).

\section{Los supuestos explícitos e implícitos de las nuevas orientaciones en las políticas sectoriales}

A continuación enumeraremos varias de las políticas reseñadas en el cuadro 1, formulando nuestros reparos a cada una de ellas.

i) Descentralización política y financiera orientada a responder a proyectos locales. Para que pueda llevarse a cabo los agentes deben tener: capacidad de organización y el peso político suficiente para que sus demandas sean escuchadas; capacidad de formular sus demandas con los formatos, contenidos y plazos requeridos, y capacidad de supervisión y control de la gestión regional. La experiencia muestra que muchos productores agrícolas carecen de estas características y que no tienen acceso a capacitación para adquirirlas. ${ }^{13}$

ii) Inversiones en infraestructura y servicios basadas en la demanda de la población beneficiaria y su cofinanciamiento. Debido a la baja densidad geográfica, la infraestructura y los servicios rurales son por lo general más caros por unidad de beneficio y por beneficiario, y varios de estos últimos tienen poca o nula capacidad de pago.

\footnotetext{
${ }^{13}$ Véase Durston (1999) en cuanto a cómo fomentar la adquisición de estas capacidades.
} 


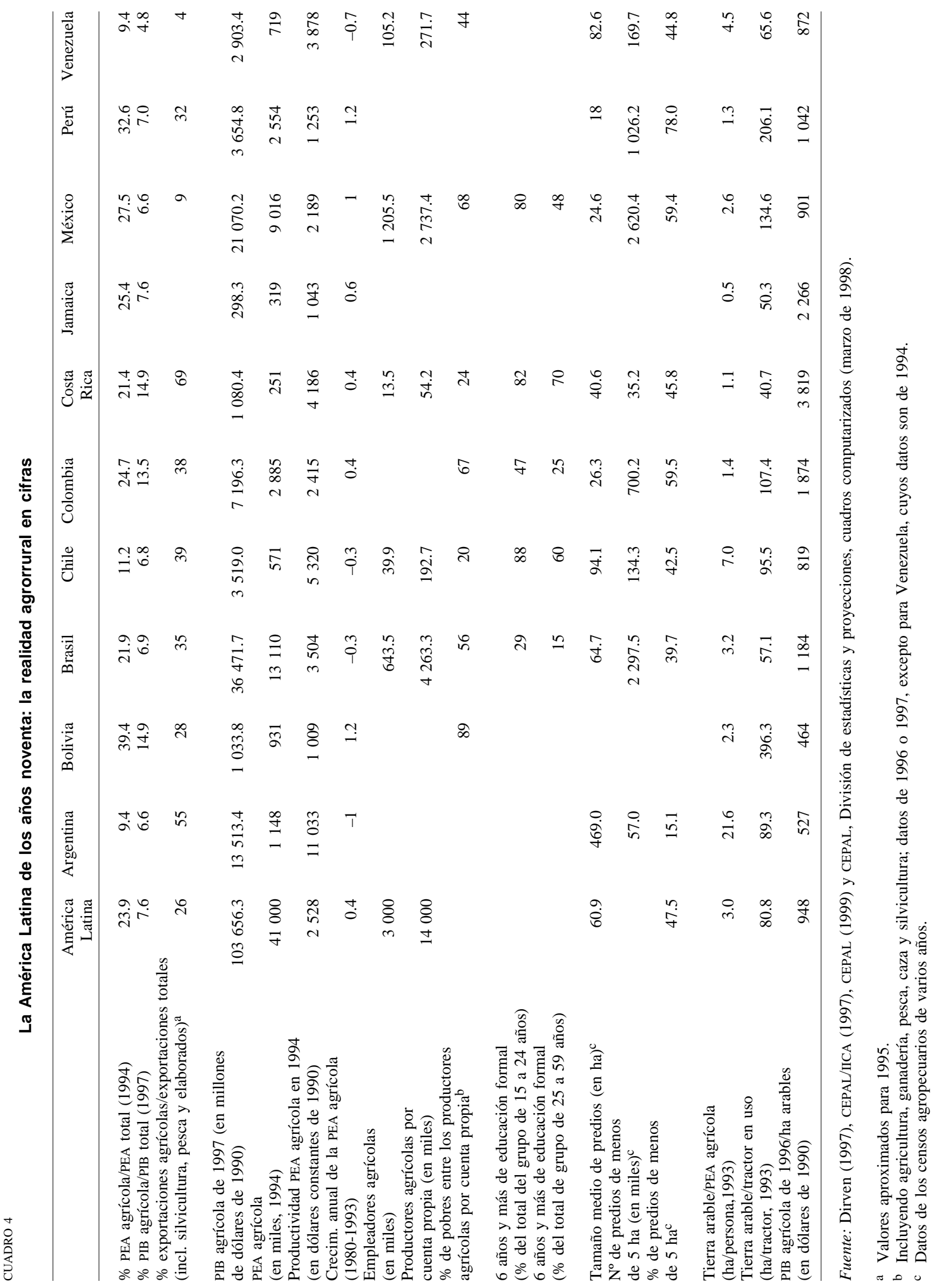


iii) Orientación hacia la demanda y el cofinanciamiento de la investigación tanto en los institutos de investigación como en las universidades. Lo que se observa es un relativo abandono de la investigación básica y una mayor orientación hacia investigaciones de más corto plazo y directamente aplicables, un desplazamiento desde los productos básicos hacia los no tradicionales y de mayor valor agregado, y una tensión entre los centros de investigación internacional que siguen orientados a la investigación de alimentos básicos y los centros nacionales de investigación, que responden a la demanda de los productores más dinámicos.

iv) Eliminación de la asistencia técnica gratuita estatal y su reemplazo por asistencia técnica privada y pagada. Esto se hace en respuesta a la demanda de los productores y después de la presentación de un proyecto a concurso cuando es acompañada de un subsidio. Los problemas surgen por falta de capacidad de pago de algunos productores; capacidad deficiente de formular en los formatos requeridos proyectos que puedan presentarse a concurso; poca divulgación y socialización de las experiencias; incumplimiento de plazos y falta de continuidad de los esfuerzos; desinterés de los consultores por llegar a lugares más lejanos o hacer proyectos más complejos y riesgosos, e insuficiente capacidad de evaluación de las propuestas y de control de los proyectos por parte de las autoridades que cofinancian o subsidian los proyectos.

v) Eliminación de líneas de crédito específicas para el sector agrícola. El sector agrícola es menos atractivo que otros sectores de la economía para las entidades crediticias debido tanto al alto riesgo intrínse-

\section{VIII}

\section{Conclusiones}

La información disponible muestra que en lo que va corrido de los años noventa, el crecimiento del producto agrícola ha estado por debajo del histórico y que las exportaciones agrícolas han crecido a un ritmo bastante inferior al de las importaciones agrícolas. Además, hay cada vez más indicaciones que apuntan a que la modernización y los aumentos en los rendimientos se concentran en un número reducido de productores agrícolas, mientras los demás estarían al margen de este avance o incluso se hallarían en retroceso. co de sus actividades (riesgo climático, fitosanitario y de fuertes fluctuaciones de precios) y los insuficientes mecanismos para aminorarlo (seguros, fondos de estabilización, ventas a futuro), como al fuerte endeudamiento de muchos agricultores y a su dispersión geográfica. Por la información parcial proveniente de varios países de la región, ${ }^{14}$ el crédito al sector agrícola habría disminuido sustancialmente a partir de las reformas, especialmente para los pequeños y medianos productores.

vi) Reemplazo de los programas de reforma agraria por compras a través del mercado de tierras, asistidas por crédito y/o subsidio para los pequeños productores. Los esfuerzos por evaluar si se están logrando los objetivos perseguidos sólo se han iniciado recientemente. Según algunos estudios ${ }^{15}$ en México y Perú (países ambos que revisaron a fondo la ley que regía el mercado de tierras a principios de los años noventa) el mercado tanto de arriendo como de compraventa de tierras es en general relativamente poco dinámico, pero tiende a la concentración de la propiedad en los tramos de propietarios medianos a grandes.

Por lo visto, se está en presencia de políticas aparentemente neutras, pero que en la realidad son a menudo muy excluyentes porque no toman en cuenta las especificidades del sector agrícola, las graves imperfecciones de varios de sus mercados y los altos costos de transacción imperantes, ni la falta de capital físico, financiero, humano y social de muchos de sus agentes. Sus resultados, sin embargo, son considerados política y moralmente justificados por constituir "respuestas a la demanda".
A nuestro juicio, el hecho de que grandes grupos de productores no participen en los procesos dinamizadores que — según los que impulsaron las refor-

\footnotetext{
${ }^{14}$ Entre otros Brasil, Costa Rica, México y Perú. (La información se basa en ponencias y documentos aún no publicados que se presentaron en el seminario El impacto de las reformas estructurales sobre la agricultura de América Latina, organizado por la CEPAL (Santiago de Chile, 25 a 27 de noviembre 1998).

${ }^{15}$ Llevados a cabo en 1997 y 1998 en virtud de un proyecto CEPAL/ FAO en el primer caso y de un proyecto CEPAL/GTZ en el segundo.
} 
mas- debían iniciarse con las reorientaciones de las políticas, se debe a falta de realismo: no se tomaron suficientemente en cuenta las peculiaridades del sector agrícola y las desventajas de sus agentes. Esta falta de repuesta de un grupo importante de agentes sería la responsable de que en las cifras agregadas el sector muestre un desempeño más bien mediocre.

¿Puede haber desarrollo rural dinámico sin esta inserción productiva? ¿Puede haber desarrollo nacional dinámico, equilibrado y equitativo con la exclusión que deriva de las políticas examinadas? ¿Cuáles son las alternativas y los costos y beneficios de las distintas opciones?

Las respuestas son difíciles, más aún por cuanto las intervenciones sectoriales y regionales tienen poca cabida en un modelo regido por los mercados.

La persistencia de la pobreza rural ha generado políticas de inversión en infraestructura y servicios (sobre todo de salud y educación) que ciertamente son necesarias y que - por las líneas de lo que han sugerido De Janvry y Sadoulet (1998) — debieran ayudar a reducir en parte los costos de transacción y, por lo tanto, permitirían que un grupo mayor de agentes se insertara lucrativamente en el mercado.

Otra respuesta ha sido dividir la población rural pobre entre los que tienen — según sus activos- perspectivas de superar su condición de pobreza a través de mejoramientos productivos, comerciales u organiza- tivos, y los que son considerados objeto (¿sujetos?) de políticas sociales. Ahora bien, mientras los que caen en esta última categoría sean una proporción relativamente pequeña de la población total o de la población de una región en particular, esta posición es sostenible desde el punto de vista económico, aunque no lo es necesariamente desde el punto de vista del desarrollo en general y menos aún del de la formación de capital humano y social. Pero, ¿qué hacer si la población que cae en esta categoría representa una alta proporción de la población total? ¿Cómo fomentar en un plazo y a un costo razonables un círculo virtuoso de actividades productivas agrícolas o no agrícolas en áreas con recursos naturales poco abundantes y con capital físico y humano poco desarrollados? ${ }^{16}$

La verdad es que aunque se contara con un marco político y conceptual que permitiese una acción más decisiva, no quedaría claro si es posible inducir este círculo virtuoso y cómo hacerlo. Urge que los próximos esfuerzos de elaboración técnica y de planteamientos concretos y prácticos busquen respuestas a estos interrogantes.

\footnotetext{
${ }^{16}$ Esta última preocupación se plasmó gracias, entre otras cosas, a las reiteradas conversaciones sobre el tema con Maximiliano Cox, Asesor Regional de la CEPAL, y también con Julio Berdegué, Director de la Red Internacional de Metodología de Investigación de Sistemas de Producción (RIMISP).
} 
APENDICE 1

\section{América Latina: Evolución de los institutos nacionales de innovación para la agricultura (INIA)}

1960: El paquete tecnológico "revolución verde" se difunde como un bien público.

1970: Aumentan las asignaciones presupuestarias a los INIA y crecen sus plantas de funcionarios.

1980: La crisis de los ochenta afecta severamente el financiamiento de los INIA. Hacia fines de la década, los efectos de la crisis y de los programas de ajuste terminan afectándolos a todos.

A la reducción de las asignaciones presupuestarias se agregan las reducciones de recursos financieros para investigación agrícola por parte de las agencias internacionales y de los países donantes.

1990: Se inician reformas en casi todos los INIA de la región, en un ambiente caracterizado por la escasez relativa de fondos para la investigación, la creciente apropiabilidad de la tecnología y el surgimiento de nuevas demandas sobre los INIA. Estas últimas aparecen principalmente como consecuencia de los fuertes procesos de urbanización (demanda de tecnologías de poscosecha), y de los procesos de apertura y desregulación (tecnologías y procesos para aumentar el valor agregado, mejorar la competitividad y desarrollar producciones no tradicionales de exportación).

En otro plano, las demandas por un mayor protagonismo del sector privado, se expresan en reformas en la organización y constitución de los INIA.

Aparecen nuevas fuentes de financiamiento como las fundaciones para la investigación y la transferencia de tecnología y los fondos concursables

Los principales INIA de la región se recuperan desde el punto de vista de las asignaciones presupuestarias fiscales y además incrementan notablemente la generación de recursos propios.
Se inicia la creación de los INIA en la mayor parte de los países de la región, y se fortalecen los ya existentes.

Los INIA tienen excelentes resultados respecto de la misión encomendada. Así lo indican todos los estudios de evaluación de impacto realizados.

Los INIA entran en crisis; en una primera etapa siguen creciendo por inercia las plantas de funcionarios mientras el financiamiento se reduce; hay menos recursos para investigación y menos recursos por investigador.

Hacia el final de la década en varios casos se reduce drásticamente el personal, incluso el más calificado, y en otros se reducen las plantas de funcionarios y las partidas de sueldos.

Las reformas son básicamente las siguientes:

a) Se establecen programas de investigación en función de la demanda de los clientes y se integran los productores y el sector privado en los consejos directivos.

b) Los programas relacionados con la pobreza y extrema pobreza y la preservación de los recursos naturales y el medio ambiente, pasan a ser prioritarios, en gran medida por demanda de las agencias internacionales.

c) En muchos INIA el desarrollo de procesos y tecnologías para mejorar la competitividad, las tecnologías poscosecha y las biotecnologías pasan a tener prioridad. Se establecen programas agroindustriales y de desarrollo de cultivos no tradicionales de exportación.

d) En la búsqueda de una mayor eficiencia en el manejo de los recursos presupuestarios, se reducen gastos considerados menos importantes y se externalizan diversos servicios.

e) Se incrementa la generación de recursos propios de los INIA a través de la venta de servicios y bienes, e incluso a través de la venta de activos.

Se regionaliza la investigación y transferencia como una forma de hacerla más eficiente y de acercarla al cliente.

Fuente: César Morales, CEPAL, sobre la base de información de los distintos INIA. 
APENDICE 2

Cambios recientes en las legislaciones de tenencia de tierras

País, ley y su propósito
Bolivia, 1996. Ley del Servicio Nacional de
Reforma Agraria o Ley INRA.

Propósito: aumentar la equidad y seguridad en la tenencia de tierras

Colombia, 1994. Ley 160, que crea el Sistema Nacional de Reforma Agraria y Desarrollo Rural Campesino.

Propósito: facilitar el acceso a tierras a los campesinos pobres.

México, 1992. Enmienda al artículo 27 de la Constitución de 1917.

Propósito: eliminar los frenos al desarrollo.

Perú, 1991, 1992 y 1995, Ley Agraria. Propósito: ampliar y fortalecer los derechos de propiedad privada.

\section{La ley en grandes líneas}

Los indígenas tienen acceso preferencial a tierras públicas.

Proceso de saneamiento de títulos a diez años plazo.

Impuesto sobre la tierra.

Los beneficiarios del programa son los responsables de negociar la compra de la tierra directamente con los propietarios.

El Estado otorga un subsidio directo de hasta $70 \%$ del precio de compra.

El $30 \%$ restante puede ser financiado mediante crédito estatal de largo plazo.

Se termina con la obligación del gobierno de entregar tierras a los que lo solicitan.

Los ejidatarios pueden pasar al status de propietario privado y vender, arrendar, dar en aparcería o hipotecar su propiedad.

Se elimina la obligación del ejidatario de trabajar su tierra personalmente.

Se mantienen límites al tamaño (100 ha de riego).

Las sociedades mercantiles pueden adquirir tierras.

Permiso de vender y gravar tierras de la reforma agraria.

Permiso para la titulación individual de tierras comunales con el acuerdo de más del $50 \%$ de los comuneros.

Se mantienen límites al tamaño (1 000 ha).

Las sociedades mercantiles pueden adquirir tierras.

\section{Fortalecimiento institucional}

Catastro y registro con administración a nivel de municipio.

Sistema de solución de problemas.

Reestructuración del INRA para adecuarse al nuevo papel de facilitar transacciones y supervigilarlas.

Creación de Tribunales Agrarios locales para resolver disputas sobre derechos de propiedad.

Creación de la Procuraduría Agraria para vigilar la correcta aplicación de la Ley.

Creación del Registro Nacional Agrario.

Programa de Certificación de Derechos Ejidales y Titulación de Solares urbanos (PROCEDE).

Proyecto especial de titulación de tierras.

Fuente: Información proporcionada por Frank Vogelgesang, basada en el Proyecto CEPAL/GTZ sobre Políticas para promover los mercados de tierras rurales. 


\section{Bibliografía}

Baer, W. y W. Maloney (1997): Neoliberalismo e distribução, Revista de economia política, vol. 17, № 3, São Paulo, Centro de Economia Política, julio-septiembre.

Banco Mundial (1996): From Vision to Action, Washington D.C. Binswanger, H. P. y K. Deininger (1997): Explaining Agricultural and Agrarian Policies in Developing Countries, Policy research working paper $\mathrm{N}^{\circ} 1765$, Washington, D.C., Banco Mundial.

CELADE (Centro Latinoamericano de Demografía) (1995): América Latina: proyecciones de población urbana-rural 1970-2025, Boletín demográfico, $\mathrm{N}^{\circ} 56$, Santiago de Chile.

(1996): América Latina: población económicamente activa 1980-2025, Boletín demográfico, $\mathrm{N}^{\circ} 57$, Santiago de Chile.

CEPAL (Comisión Económica para América Latina y el Caribe) (1995): Reformas económicas en América Latina: una síntesis de la experiencia en once países, LC/R.1606, Santiago de Chile.

(1997a): Estrategia de apertura comercial: planteo teórico y evidencias empíricas, LC/R.1732, Santiago de Chile.

(1997b): La participación organizada en el desarrollo agro-rural del Paraguay, LC/R.1765, Santiago de Chile.

(1999): Panorama social 1998, LC/G. 2050-P, Santiago de Chile.

CEPAL/IICA (Instituto Interamericano de Cooperación para la Agricultura) (1997): Panorama de la agricultura de América Latina y el Caribe en las últimas décadas (indicadores de comportamiento en cuadros y gráficos), LC/L.1102, Santiago de Chile.

Colombia, Ministerio de Agricultura (1993): El balance del sector agropecuario en 1993: la política de reactivación y sus frutos, Santafé de Bogotá, diciembre.

De Janvry, A. y E. Sadoulet (1993): Market, State, and civil organizations in Latin America, Beyond the debt crisis: the context for rural development, World Development, vol. 21, $\mathrm{N}^{\circ}$ 4, Oxford, Reino Unido, Pergamon Press.

(1998): Smallholder integration into markets: determinants of entry and supply response, presentado al Tercer Simposio Latinoamericano sobre Investigación y Extensión en Sistemas Agropecuarios (IESA-AL-III): Nuevos enfoques para la su- peración de la pobreza rural y para el desarrollo de capacidades locales, Lima, 19 al 21 de agosto.

Dirven, M. (1997): El empleo agrícola en América Latina y el Caribe: pasado reciente y perspectivas, Desarrollo productivo $\mathrm{N}^{\circ}$ 43, LC/G.1961, Santiago de Chile, CEPAL.

Durston, John (1999): Construyendo capital social comunitario. Una experiencia de empoderamiento rural en Guatemala, LC/L.1177, Naciones Unidas.

Edwards, S. (1997): El mal desempeño de las economías latinoamericanas, Estudios Públicos, No 67 , Santiago de Chile, Centro de Estudios Públicos (CEP).

FAO (Organización de las Naciones Unidas para la Agricultura y la Alimentación) (1994): Anuario. Producción 1994, Roma. (1996): Estado mundial de la agricultura y la alimentación, Roma.

Gómez Oliver, L. (1994): La política agrícola en el nuevo estilo de desarrollo latinoamericano, Santiago de Chile, Organización de las Naciones Unidas para la Alimentación y la Agricultura (FAO).

IICA/Instituto Internacional de Investigaciones sobre Políticas Alimentarias (1996): Política agrícola: la búsqueda de la competitividad, sostenibilidad y equidad, Santafé de Bogotá, Unión Gráfica Ltda.

Klein, E. (1992): El empleo rural no agrícola en América Latina, Documento de trabajo, № 364, Santiago de Chile, Programa Regional del Empleo para América Latina y el Caribe (PREALC).

Krueger A. O., M. Schiff y A. Valdés (1990): Economía política de las intervenciones de precios agrícolas en América Latina, San Francisco, California, Banco Mundial.

Peres, W., coord. (1998): Grandes empresas y grupos industriales latinoamericanos, México D.F., Siglo Veintiuno Editores.

Ramos, J. (1997): Un balance de las reformas estructurales neoliberales en América Latina, Revista de la CEPAL, $\mathrm{N}^{\circ}$ 62, LC/G.1969-P, Santiago de Chile, CEPAL.

Schejtman, A. (1982): Economía campesina y agricultura empresarial: tipología de productores del agro mexicano, México D.F., Siglo Veintiuno Editores. 